\title{
Response to letter commenting on published paper: Adding abiraterone to androgen deprivation therapy in men with hormone-sensitive prostate cancer: A systematic review and meta-analysis
}

Larysa H.M. Rydzewska*

larysa.rydzewska@ucl.ac.uk

Sarah Burdett

Claire L. Vale

Mahesh K.B. Parmar

Jayne F. Tierney

MRC Clinical Trials Unit at UCL, London, UK

*Corresponding author: Meta-analysis Group, MRC Clinical Trials Unit at UCL, 90 High Holborn, $2^{\text {nd }}$ Floor, London, WC1V 6LJ, UK.

To the Editor,

Response to Christopher J.D. Wallis, Zachary Klaassen's Letter to the Editor re: Rydzewska LHM, Burdett S, Vale CL, Clarke NW, Fizazi K, Kheoh $T$ et al. Adding abiraterone to androgen deprivation therapy in men with metastatic hormone-sensitive prostate cancer: a systematic review and meta-analysis. Eur J Cancer. 2017; 84:88-101. https://doi.org/10.1016/j.ejca.2017.07.003

Our systematic review and meta-analysis was conducted using FAME (Framework for Adaptive Meta-analysis, [1]). This is a prospective approach that takes all eligible trials into account and is responsive to emerging trial results. Thus, we would like to make clear that the review was initiated well in advance of the two trial results being known, and we anticipated that a metaanalysis of these trials would provide both a timely and sufficiently reliable result. It would have been near impossible to predict or expect that the individual trials would produce such 'overwhelmingly positive' or 'remarkably consistent' results.

As Wallis et al. point out, the meta-analysis has improved the precision of the estimate of effect on survival. However, by using FAME, working closely with the trial teams, and requesting additional analyses and results, we have been able to do much more 'to advance our understanding of the role of abiraterone in men with metastatic hormone-sensitive prostate cancer':

- $\quad$ By obtaining additional unreported progression-free survival (PFS) analyses for STAMPEDE metastatic (M1) patients, we have been able to look at the consistency of effect across the two trials and to provide the best estimate of the size of the PFS effect.

- $\quad$ By obtaining subgroup analyses just for STAMPEDE M1 patients, and additional analyses of LATITUDE, we have been able to investigate whether the effects of abiraterone on survival and PFS are consistent across different types of men, with greater power than the individual trials and using analyses that are not usually possible with aggregate data [2].

- In particular, we showed that the difference in effect by age group seen in STAMPEDE remains in the pooled analysis and requires further investigation with individual participant data (IPD).

- By collecting consistently defined G3-4 and G5 acute toxicity for both trials, and just for STAMPEDE M1 patients, we have provided the first formal analysis of these outcomes, both within and across trials. We found an increase in serious acute cardiac, vascular and hepatic side-effects with abiraterone, but no excess of deaths.

- By obtaining up-to date information on the PEACE-1 trial, we were able to place the results in the context of all the potential evidence.

We very much agree that there is now a need to compare the relative efficacy of different treatments, including docetaxel and abiraterone, and this review feeds into a network meta-analysis based on aggregate data [3]. Our results suggest that adding abiraterone to androgen deprivation therapy (ADT) is likely to be the most effective, and docetaxel plus ADT the second most effective treatment in improving survival in men with metastatic hormone-sensitive prostate cancer, although some uncertainty remains about the absolute difference in survival benefit between these two treatments and how trial and patient variability impacts on these results. Our ongoing collection of IPD will help us determine more definitively which therapies work best, and for which men.

\section{Conflict of interest statement}

None declared. 


\section{References}

[1] J.F. Tierney, C.L. Vale, S. Burdett, D. Fisher, L.H.M. Rydzewska and M.K.B. Parmar, Timely and reliable evaluation of the effects of interventions: a framework for adaptive meta-analysis (FAME), Trials 18 (Suppl. 1), 2017, P351.

[2] D.J. Fisher, J.R. Carpenter, T.P. Morris, S.C. Freeman and J.F. Tierney, Meta-analytical methods to identify who benefits most from treatments: daft, deluded, or deft approach?, BMJ 356, 2017, j573.

[3] C.L. Vale, D.J. Fisher, I.R. White, J. Carpenter, S. Burdett, N.W. Clarke et al. What is the optimal systemic treatment for men with metastatic, hormone- naive prostate cancer? A STOPCAP systematic review and network meta-analysis. Ann Oncol. 2018 (Accepted Manuscript: https://doiorg/101093/annonc/mdy071) 